
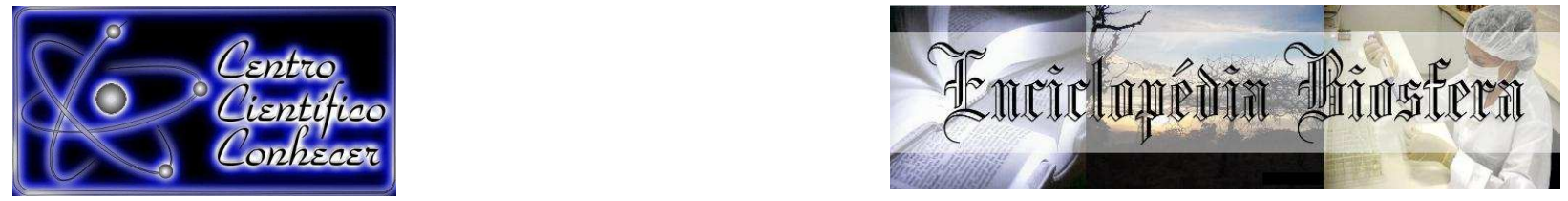

\title{
LORANTHACEAE JUSS. NO ACERVO DO HERBÁRIO DA AMAZÔNIA MERIDIONAL, ALTA FLORESTA, MATO GROSSO
}

Dennis Rodrigues da Silva ${ }^{1,6}$, Célia Regina Araújo Soares-Lopes ${ }^{2,6}$, David de Souza Verão $^{3,6}$, Lucirene Rodrigues ${ }^{4,6}$, Ricardo da Silva Ribeiro ${ }^{5,6}$

1Mestrando do Programa de Pós-Graduação Stricto Sensu em Biodiversidade e Agroecossistemas Amazônicos, Universidade do Estado de Mato Grosso (drsilva.af@gmail.com)

2Professora Adjunto VI, Faculdade de Ciências Biológicas e Agrárias, Universidade do Estado de Mato Grosso, Campus Universitário de Alta Floresta

${ }^{3}$ Bacharel em Engenharia Florestal, Universidade do Estado de Mato Grosso

${ }^{4}$ Mestre em Ecologia e Conservação, Universidade do Estado de Mato Grosso, Campus Universitário de Nova Xavantina - MT

${ }^{5}$ Graduando em Ciências Biológicas, Universidade do Estado de Mato Grosso

${ }^{6}$ Herbário da Amazônia Meridional, UNEMAT, Alta Floresta

Recebido em: 08/09/2015 - Aprovado em: 14/11/2015 - Publicado em: 01/12/2015 DOI: http://dx.doi.org/10.18677/Enciclopedia_Biosfera_2015_252

\section{RESUMO}

Loranthaceae é considerada a família com maior diversidade de espécies dentre as plantas hemiparasitas. Distribui-se por todo território brasileiro nos diferentes domínios fitogeográficos, porém são poucos os relatos de ocorrência da espécie para a Amazônia Meridional. Desta forma, o presente trabalho teve como objetivo apresentar uma listagem para as espécies de Loranthacae depositadas no Herbário da Amazônia Meridional - HERBAM, Alta Floresta, Mato Grosso. A validação das identificações dos espécimes de Loranthaceae ocorreu em agosto de 2015 pelos autores com base nos materiais revisados por especialista, nas revisões taxonômicas recentes e imagens disponibilizadas por herbários virtuais. Loranthaceae está representada por 13 espécies distribuídas em cinco gêneros. A espécie Tripodanthus acutifolius (Ruiz \& Pav.) Tiegh. é o primeiro registro genérico para o domínio amazônico. O gênero com maior riqueza específica foi Psittacanthus. O presente trabalho possui informações importantes sobre a Flora do Mato Grosso e, portanto, para atualização da "Lista de Espécies da Flora do Brasil".

PALAVRAS-CHAVE: Distribuição; Floresta Amazônica, hemiparasitas, Tripodanthus acutifolius

\section{LORANTHACEAE JUSS. IN THE HERBÁRIO DA AMAZÔNIA MERIDIONAL COLLECTION, ALTA FLORESTA, MATO GROSSO}

\begin{abstract}
Loranthaceae is considered the plant family with the greatest diversity of species among mistletoes plants. It is distributed throughout Brazil in different phytogeographical domains, but there are few reports about occurrence of family to Southern Amazon. Thus, this study aimed to present a listing to Loranthaceae
\end{abstract}


species deposited in Herbário da Amazônia Meridional - HERBAM, Alta Floresta, Mato Grosso. The validation of identifications of Loranthaceae specimens occurred in August of 2015 by the authors based on the materials specialist reviewed, in recent taxonomic revisions and available pictures by virtual herbarium. Loranthaceae is represented by 13 species distributed in five genera. The Tripodanthus acutifolius (Ruiz \& Pav.) species is the first genera record to Amazonian domain. The genus with the highest specific richness was Psittacanthus. This study has informations about Mato Grosso Flora and, therefore to update the "Lista de Espécies da Flora do Brasil".

KEYWORDS: Distribution, Amazonian forest, mistletoes, Tripodanthus acutifolius

\section{INTRODUÇÃO}

Loranthaceae Juss. compreende quase 1.000 espécies distribuídas em 73 gêneros, sendo considerada a mais representativa dentre as famílias de plantas hemiparasitas estabelecidas sobre árvores ou arbustos hospedeiros, conhecidas como ervas-de-passarinho, e possuem distribuição predominantemente pantropical (NICKRENT, 2011; SOUZA \& LORENZI, 2012; PEREIRA, 2014). As principais características morfológicas da família estão pautadas na forma de vida, sendo ervas ou arbustos hemiparasitas cujo sistema radicular modificado é denominado haustório, na ausência de catafilos e na presença de folhas opostas (raramente alternas), sem estípulas; flores maiores que $5 \mathrm{~mm}$, diclamídeas, normalmente andróginas, quando unissexuais são dioicas, ovário ínfero; fruto baga ou drupa com uma semente (CRONQUIST, 1981; JUDD et al., 2009; NICKRENT et al., 2010; CAIRES, 2012; DETTKE \& WAECHTER, 2014).

O termo erva-de-passarinho deve-se a forte relação de mutualismo dessas plantas, principalmente, com as aves. As ervas-de-passarinho fornecem alimento por meio de seus frutos que, após passarem pelo sistema digestor das aves, as quais realizam a dispersão das sementes regurgitando ou defecando nas hastes ou nos ramos das plantas hospedeiras (DAVIDAR, 1983; MARTíNEZ DEL RIO et al., 1996).

As ervas-de-passarinho são conhecidas principalmente pela ação nociva que provocam aos hospedeiros e pelo prejuízo a silvicultura, agricultura (principalmente nas plantações de cítricos) e também diminuindo a qualidade da arborização urbana (TORRES \& MONTILLA, 1997; ROTTA, 2001; LEAL et al. 2006; MATHIASEN et al., 2008; WHITE et al. 2011, PEREIRA, 2014).

$\mathrm{Na}$ entrada do século XXI, entretanto, estudos começaram a mostrar que essas espécies de plantas podem assumir papel chave nos ecossistemas, proporcionando abrigo para ninhos de aves, fornecendo recurso alimentar para aves, diferentes grupos de insetos (GUERRA \& MARINI, 2002; COONEY \& WATSON, 2005; COONEY et al., 2006; CAIRES, et al., 2009; VICECONTE \& MAIA, 2009; ARRUDA et al., 2012) e pequenos mamíferos como os marsupiais Dromiciops gliroides Thomas, 1894 e Gracilinanus agilis Burmeister, 1854 (GARCÍA et al., 2009; CAMARGO et al., 2011).

Estudos farmacológicos e da medicina popular também têm atribuído importância às espécies de erva-de-passarinho, as espécies do gênero Struthanthus Mart. tem recebido destaque principal (VIEIRA et al., 2005; DIBONG et al., 2009; FREIRE et al., 2011). Trabalhos como estes surgem a partir de amostragens que divulgam as espécies ocorrentes em uma determinada região. 
FORZZA et al. (2012) relatam que o Estado de Mato Grosso, tem a flora subamostrada. As amostragens de Loranthaceae para o Mato Grosso que se tem conhecimento estão concentradas nas áreas do Pantanal e do Cerrado cujos trabalhos estão, em sua maioria, restringidos a monografias e trabalhos divulgados em anais de eventos (CARNIELLO et al., 1995; SANTOS-SILVA, 2001; SANTOSSILVA, 2002; SANTOS-SILVA \& CARNIELLO, 2002; SANTANA et al., 2007; SANTOS-SILVA \& CARNIELLO, 2008; SILVA, 2009; SILVA, et al., 2009; SILVA, et al., 2010), tendo portanto, desconhecimento de trabalhos sobre Loranthaceae com foco na Amazônia mato-grossense, onde SPLETOZER et al. (2014) e RODRIGUES et al. (2014) alertam que as paisagens vêm sofrendo com o uso e a ocupação do solo, através do avanço das pastagens e o aumento da fragmentação da vegetação.

Para contribuir com a diminuição das lacunas de conhecimento sobre a flora da região norte mato-grossense, foi criado em 2007, o Herbário da Amazônia Meridional (HERBAM), no Campus Universitário de Alta Floresta, Universidade do Estado de Mato Grosso. De acordo com IGANCI \& MORIN (2012) é imensurável a importância das coleções botânicas como fonte de dados para o conhecimento da flora, elaboração de planos de conservação e preservação de espécies, bem como a documentação da distribuição das espécies vegetais.

Portanto, as plantas coletadas na região da Amazônia Meridional depositadas no HERBAM têm contribuído significativamente para ampliar o conhecimento sobre a flora do Mato Grosso registrando novas ocorrências para Estado (SOARESLOPES et al., 2014; FERNANDES et al., 2015; PETINI-BENELLI et al., 2015) e também novas espécies para a ciência (VANDERPLANK \& ZAPPI, 2011; CHIRON \& PETINI-BENELLI, 2012; PENNINGTON \& CLAKSON, 2013; FRISBY \& HIND, 2014; PETINI-BENELLI \& SOARES-LOPES, 2015). Este trabalho teve como objetivo apresentar uma listagem das espécies de Loranthaceae depositadas na coleção do Herbário da Amazônia Meridional - HERBAM e informações sobre sua distribuição na região.

\section{Área de Estudo}

\section{MATERIAL E MÉTODOS}

O estudo foi realizado a partir da compilação dos Bancos de Dados do Herbário da Amazônia Meridional - HERBAM, situado no Centro de Pesquisas em Biodiversidade da Amazônia Meridional - UNEMAT, na MT 208, KM 146, S/N Jardim Tropical, Alta Floresta, Mato Grosso, relacionadas aos resultados de coletas realizadas na região norte do Estado de Mato Grosso, nos municípios de Alta Floresta, Colíder, Nova Canaã do Norte, Novo Mundo, Paranaíta e Santa Cruz do Xingu (Mato Grosso) e Jacareacanga (Pará), (Figura 1). 


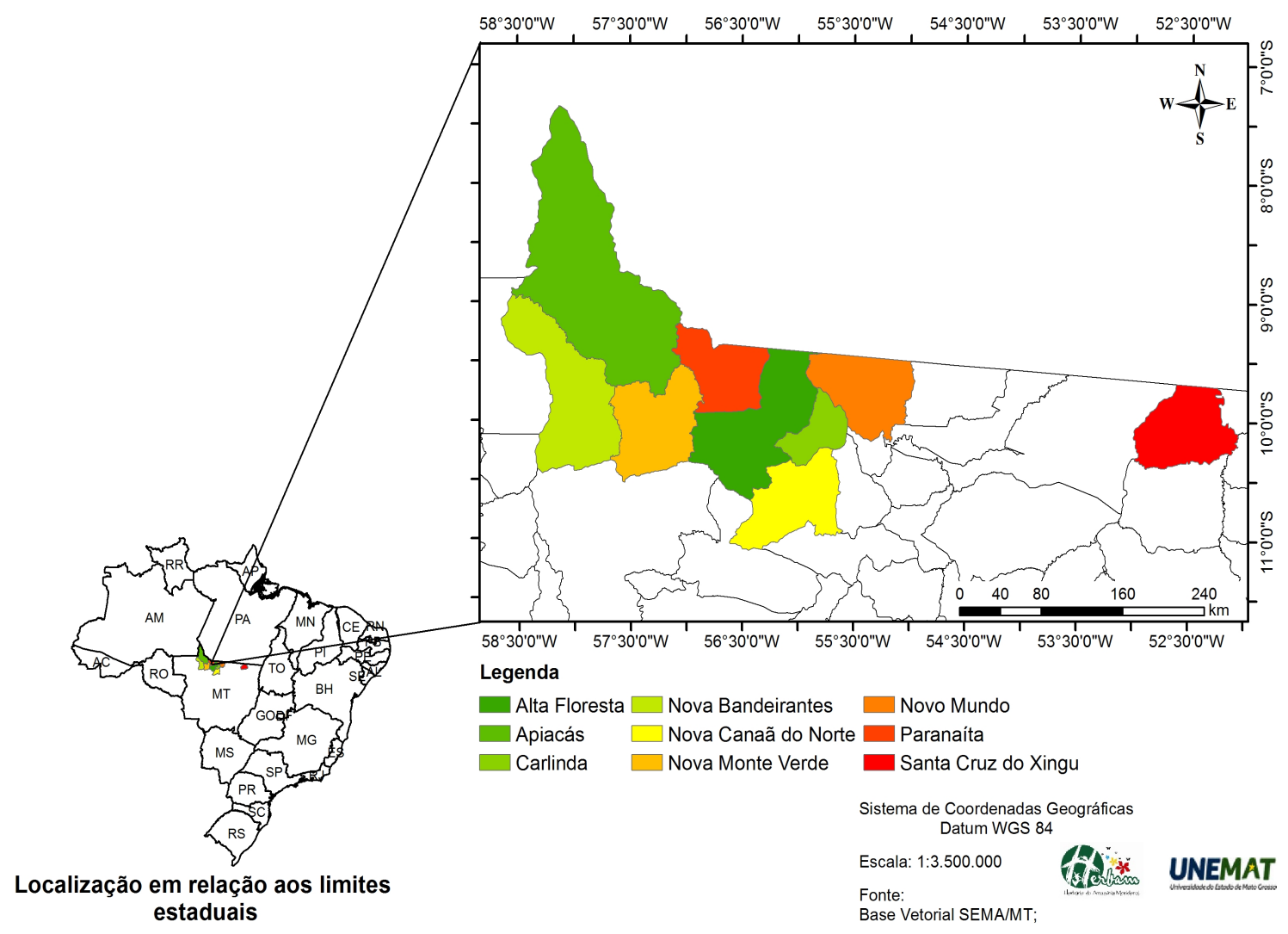

FIGURA 1. Municípios com amostras botânicas de Loranthaceae depositadas no Herbário da Amazônia Meridional, Alta Floresta, Mato Grosso.

Os municípios com ocorrência de Loranthaceae registradas na coleção do HERBAM fazem parte da região conhecida como arco do desmatamento ou Portal da Amazônia, cujo clima é quente e úmido, com temperatura média de $24^{\circ} \mathrm{C}$ ao ano e pluviosidade média superior a $2.400 \mathrm{~mm}$ ao ano, destacando-se uma estação seca bem definida de até cinco meses. Conforme Koopen, o tipo climático Am e Aw (ALVARES et al., 2013). Dentre os tipos de solos encontrados estão o Latossolos vermelho-escuro, e Plintossolos apresentados sobre os relevos denominados como depressão do Norte de Mato Grosso, Planaltos Residuais, Depressão da Chapada do Cachimbo, Planalto do Apiacás e Planalto dos Parecis (SEPLAN, 2014). A vegetação é composta por Floresta Ombrófila Densa e Aberta, Floresta Estacional Semidecidual, Decidual, Perenifólia, Campinarana e Campos Rupestres (IVANAUSKAS et al., 2008; SASAKI et al., 2010; ZAPPI et al., 2011; ALMEIDA et al., 2014).

Levantamento florístico - O material botânico coletado é proveniente das expedições realizadas entre 2006 e 2015, nos parques estaduais do Cristalino (PEC) e do Xingu (PEX), nos empreendimentos hidrelétricos das usinas: UHE São Manoel, UHE Colider, UHE Teles Pires e Complexo Hidrelétrico Apiacás, e ao longo de pontos de pesca utilizados pela Pousada Mantega no Rio Teles Pires. Os procedimentos de coleta e herborização foram realizados de acordo com FIDALGO \& BONONI (1989) e IBGE (2012). Parte do material depositado no HERBAM, foi identificado por especialista (Claudenir Simões Caires), e parte foi identificado pelos autores do presente trabalho utilizando chave (Caires, 2012) e comparando com o material já depositado no herbário identificado pelo especialista anteriormente. A 
lista das espécies foi organizada considerando APG III (CHASE \& REVEAL, 2009) como sistema de classificação. A validação dos nomes científicos das espécies e dos autores das espécies foi realizada conferindo na Lista de Espécies da Flora do Brasil (DETTKE \& CAIRES, 2015). A Lista de Espécies da Flora do Brasil também foi utilizada para sistematizar a distribuição geográfica das espécies, bem como a ocorrência das mesmas nos domínios fitogeográficos brasileiros.

\section{RESULTADOS E DISCUSSÃO}

No Herbário da Amazônia Meridional encontram-se depositados 54 espécimes de Loranthaceae agrupados em cinco gêneros e 13 espécies coletadas na região do arco do desmatamento (Quadro 1 e Figura 2), correspondendo a 17,8\% das espécies de Loranthaceae citadas para a Amazônia brasileira por DETTKE \& CAIRES (2015). O gênero Psittacanthus Mart. está representado por seis espécies, Oryctanthus (Griseb.) Eichler por três espécies, Struthanthus Mart. por duas espécies e Passovia H.Karst. e Tripodanthus (Eichler) Tiegh. estão representados por uma espécie cada.

Dentre as espécies de Loranthaceae (ver distribuição na Figura 2) apresentadas no presente trabalho, a ocorrência de Oryctanthus spicatus não é constatada para o estado de Mato Grosso de acordo com a Lista de espécies da Flora do Brasil (DETTKE \& CAIRES, 2015), mas não foi considerada como nova ocorrência devido a mesma espécie já ter sido citada para o Estado por CAIRES (2012). Por outro, lado a presença de Tripadanthus acutifolius representa o primeiro registro do gênero para o domínio fitogeográfico amazônico, pois só era conhecida a ocorrência de Tripodanthus nos domínios da Caatinga, do Cerrado, da Mata Atlantica e dos Pampas.

De acordo com DETTKE \& CAIRES (2015) Psittacanthus dentatus é uma espécie endêmica do Brasil, com ocorrência restrita à Amazônia, no Pará e no Mato Grosso. Ocorre em áreas de vegetação florestal densa e alta, sobre os interflúvios, sem influência da cheia dos rios durante a estação chuvosa. Esta espécie foi coletada unicamente na área do Parque Estadual do Cristalino, que foi criado em junho de 2000 pelo Decreto 1.471/00 que foi sancionado pela Lei Estadual 7.518/01 de 28 de setembro de 2001. O parque contribui com a consolidação e o fortalecimento do Corredor de Áreas Protegidas, a região do Arco do Desmatamento (ICV, 2003).

O gênero Psittacanthus Mart. tem maior destaque no herbário por ser 0 gênero de maior riqueza da família Loranthaceae no neotrópico. Conforme ARRUDA et al. (2012), Psittacanthus com 42 espécies é o gênero com maior representatividade no Brasil, com grande diversidade na Amazônia. Suas principais características são as folhas opostas, flores arquitetadas nos racemos terminais ou axilares em díades ou tríades (DETTKE \& WAECHTER, 2014).

Oryctanthus (Griseb.) Eichler, o segundo gênero mais representativo no acervo, é um gênero com 11 espécies reconhecidas, com distribuição principalmente continental. Do Sul do México atravessa a América Central, passa pelos Andes na Colômbia, Equador e Peru e pelas terras baixas do Suriname e Brasil (CAIRES, 2012). No território nacional são conhecidas quatro espécies, todas, presentes no domínio amazônico (DETTKE \& CAIRES, 2015). É caracterizada pela presença de raízes epicorticais, nascidas na base da planta, espigas congestas e carnosas, flores bissexuais, pólen com três depressões circulares (CAIRES, 2012). 
O gênero Struthanthus Mart., terceiro mais representado no herbário, possui de 60 a 70 espécies em todo o mundo, ocorrendo no neotrópico, do México até a porção central da Argentina e do Uruguai (ABBIATTI, 1946; RIZZINI, 1956 citados por DETTKE \& WAECHTER, 2014). Para o Brasil ARRUDA et al. (2012) relata a presença de 56 espécies deste gênero e, recentemente, DETTKE \& CAIRES (2015) listam 53 espécies de Struthanthus para todo Brasil. Contudo, ambos os autores concordam que o gênero é mais diverso nos Biomas Mata Atlântica, Cerrado e Amazônia, respectivamente.

QUADRO 1. Espécies, domínios fitogeográficos e distribuição geográfica das espécies de Loranthaceae depositadas no Herbário da Amazônia Meridional - HERBAM, Alta Floresta, Mato Grosso. Dom. Fitog.=Domínio Fitogeográfico ( $\mathrm{AM}=$ =Amazônia, $\quad \mathrm{CA}=$ Caatinga, $\quad \mathrm{CE}=$ Cerrado, $\mathrm{MA}=$ Mata Atlântica, $\mathrm{PA}=$ Pantanal, $\mathrm{PP}=\mathrm{Pampa}$ ); Dist. Geog.=Distribuição Geográfica (DETTKE \& CAIRES, 2015).

\begin{tabular}{|c|c|c|c|}
\hline Espécies & $\begin{array}{c}\text { Dom. } \\
\text { Fitog. }\end{array}$ & Dist. Geog. & HERBAM \\
\hline $\begin{array}{c}\text { Oryctanthus alveolatus } \\
\text { (Kunth) Kuijt }\end{array}$ & AM & $\begin{array}{c}\text { Norte: AC, AM, AP, PA, RO, RR; } \\
\text { Nordeste: MA; Centro-Oeste: MT }\end{array}$ & 3996 \\
\hline $\begin{array}{c}\text { Oryctanthus florulentus } \\
\text { (Rich.) Tiegh. }\end{array}$ & AM & $\begin{array}{c}\text { Norte: AC, AM, AP, PA, RO, RR, TO; } \\
\text { Nordeste: MA, PI; Centro-Oeste: MT }\end{array}$ & 3158 \\
\hline $\begin{array}{c}\text { Oryctanthus spicatus } \\
\text { (Jacq.) Eichler }\end{array}$ & AM & Norte: AC, AM, AP, PA & 2683 \\
\hline $\begin{array}{c}\text { Passovia pedunculata } \\
\text { (Jacq.) Kuijt }\end{array}$ & AM, CE & $\begin{array}{c}\text { Norte: AC, AM, AP, PA, RO, RR, TO; } \\
\text { Nordeste: BA, MA, PI; Centro-Oeste: GO, } \\
\text { MT; Sudeste: MG }\end{array}$ & 3160 \\
\hline $\begin{array}{c}\text { Psittacanthus acinarius } \\
\text { (Mart.) Mart. }\end{array}$ & $\begin{array}{c}\text { AM, CA, } \\
\text { CE, PA }\end{array}$ & $\begin{array}{c}\text { Nordeste: AC, AM, PA, RO, RR, TO; } \\
\text { MS, MT; Centro-Oeste: DF, GO, }\end{array}$ & 2567 \\
\hline $\begin{array}{c}\text { Psittacanthus cordatus } \\
\text { (Hoffmanns.) G.Don }\end{array}$ & $\begin{array}{c}\text { AM, CE, } \\
\text { MA, PA }\end{array}$ & $\begin{array}{c}\text { Norte: AM, PA, RO, TO; Nordeste: BA, } \\
\text { CE, PB, PE, PI, RN, SE; Centro-Oeste: } \\
\text { GO, MS, MT; Sudeste: MG, RJ, SP }\end{array}$ & 9001 \\
\hline $\begin{array}{c}\text { Psittacanthus } \\
\text { cucullaris (Lam.) } \\
\text { G.Don }\end{array}$ & AM, CE, & $\begin{array}{c}\text { Norte: AC, AM, AP, PA, RO, TO; } \\
\text { Nordeste: MA; Centro-Oeste: MT }\end{array}$ & 1392 \\
\hline $\begin{array}{c}\text { Psittacanthus dentatus } \\
\text { Kuijt }\end{array}$ & AM & Norte: PA; Centro-Oeste: MT & 1600 \\
\hline $\begin{array}{c}\text { Psittacanthus } \\
\text { peronopetalus Eichler }\end{array}$ & AM & Norte: AC, AM, PA, RO; Centro-Oeste: \\
MT & 6417 \\
\hline $\begin{array}{c}\text { Psittacanthus robustus } \\
\text { (Mart.) Mart. }\end{array}$ & $\begin{array}{c}\text { AM, CA, } \\
\text { CE, MA, } \\
\text { PA }\end{array}$ & $\begin{array}{c}\text { Nordeste: BM, MP, PA, RO, RR, TO; } \\
\text { Oeste: DF, GO, MS, MT; Sudeste: MG, } \\
\text { RJ, SP }\end{array}$ & 10395 \\
\hline $\begin{array}{c}\text { Struthanthus } \\
\text { orbicularis (Kunth) } \\
\text { Blume }\end{array}$ & AM & Norte: AM, AP; Centro-Oeste: MT & 831 \\
\hline $\begin{array}{c}\text { Struthanthus } \\
\text { syringifolius (Mart.) } \\
\text { Mart. }\end{array}$ & $\begin{array}{c}\text { AM, CA, } \\
\text { CE, MA }\end{array}$ & $\begin{array}{c}\text { Norte: AC, AM; Nordeste: AL, BA, CE, PB, } \\
\text { PE, SE; Centro-Oeste: GO, MT; Sudeste: } \\
\text { ES, MG, RJ }\end{array}$ & 2250 \\
\hline $\begin{array}{c}\text { Tripodanthus } \\
\text { acutifolius (Ruiz \& } \\
\text { Pav.) Tiegh. }\end{array}$ & $\begin{array}{c}\text { CA, CE, } \\
\text { MA, PP }\end{array}$ & $\begin{array}{c}\text { Nordeste: BA, CE, PE; Centro-Oeste: DF, } \\
\text { GO, MT; Sudeste: ES, MG, RJ, SP; Sul: } \\
\text { PR, RS, SC }\end{array}$ & 2141 \\
\hline
\end{tabular}




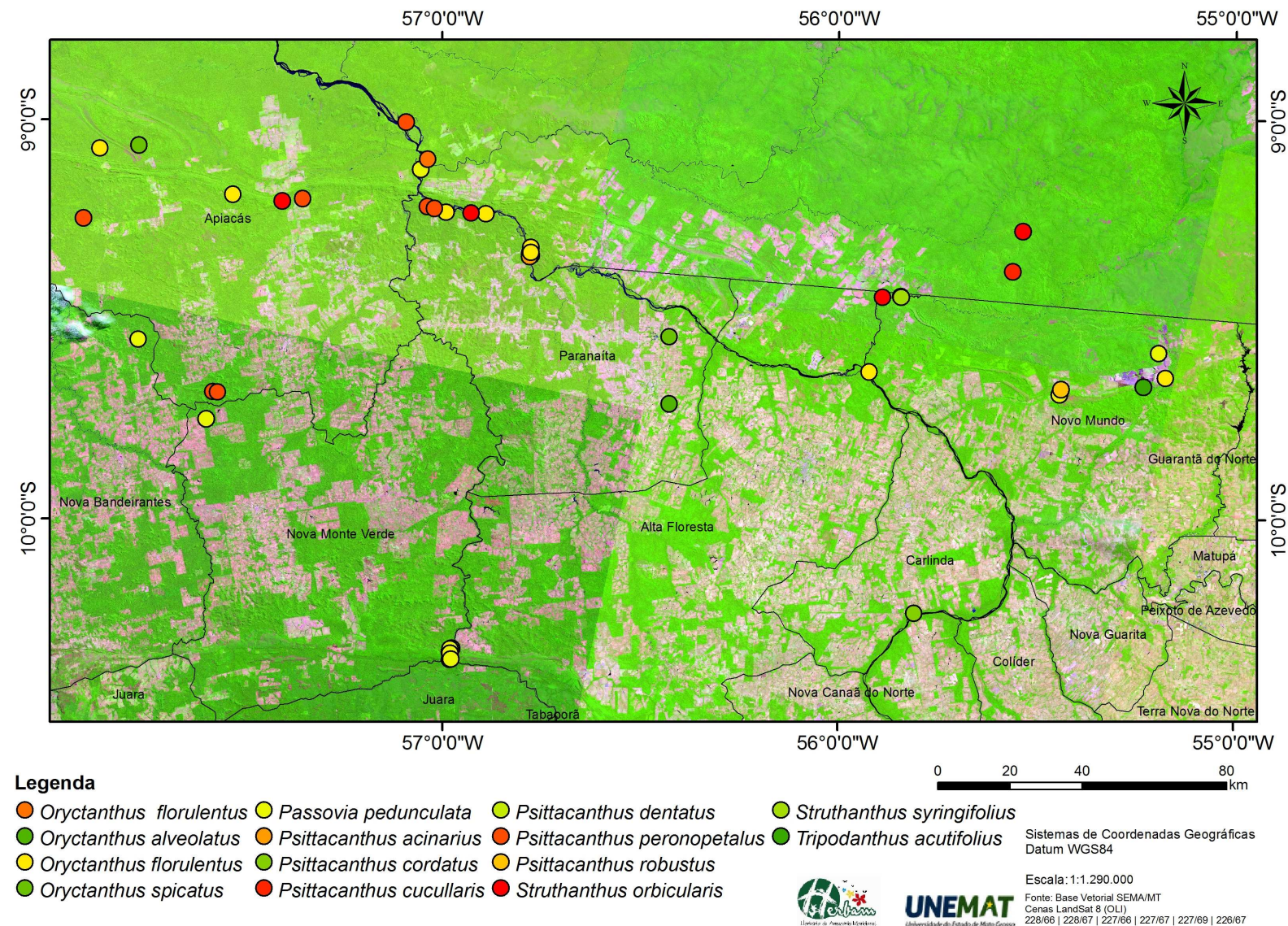

FIGURA 2. Distribuição das treze espécies de Loranthaceae coletadas na região do arco do desmatamento e depositadas no Herbário da Amazônia Meridional.

Chave para os gêneros de Loranthaceae da Amazônia Meridional (Adaptado de CAIRES, 2012).

1 Inflorescência constituída em mônades

2 Flores bissexuais; raízes epicorticais presentes

3 Espigas congestas e carnosas; pólen com 3 depressões.......Oryctanthus

3' Racemos ou espigas laxas; pólen sem depressões

2' Flores unissexuais; raízes epicorticais ausentes

1 ' Inflorescência constituída em díades ou tríades

4 Hemiparasitas terrestres raro aéreas; arbóreas a arbustivas; flores amarelodouradas; brácteas foliáceas

4' Hemiparasitas sempre aéreas; herbáceas, lianescentes ou arbustivas; flores brancas cremes a amareladas; brácteas escamiformis

5 Flores > 1,5 cm de comprimento; endosperma ausente

6 Anteras dorsifixas, versáteis Psittacanthus

6' Anteras basifixas não versáteis

5' Flores < 1,5 cm de comprimento; endosperma presente

7 Flores pediceladas Tripodanthus 7' Flores sésseis 
8 Filetes achatados e lateralmente escavados; anteras basifixas. Passovia

8' Filetes cilíndricos não escavadas; anteras
dorsifixas.....................................................Struthanthus

\section{CONCLUSÃO}

O trabalho registra a primeira ocorrência do gênero Tripodanthus na Amazônia e reforça a citação de ocorrência da espécie Oryctanthus spicatus para o estado, sendo uma importante contribuição para a flora matogrossense e para 0 domínio amazônico.

É importante evidenciar a importância das áreas de preservação dentro dos parques presentes na região da Amazônia Meridional para a manutenção de espécies endêmicas do Brasil. Trabalhos como este devem continuar sendo realizados dentro do estado, uma vez que o mesmo possui três biomas com lacunas de conhecimento sobre a flora do Mato Grosso a serem preenchidas.

\section{REFERENCIAS}

ABBIATTI, D. Las Lorantáceas argentinas. Revista del Museo de La Plata, Botánica. v. 28, p. 1-110, 1946.

ALMEIDA, E. A.; SOARES-LOPES, C. R. A.; RODRIGUES, L.; SIMÃO, S. S.; FERNANDES, J. M. Estrutura fitossociológica de floresta estacional decidual submontana e floresta ombrófila aberta submontana em Alta Floresta, Mato Grosso. Enciclopédia Biosfera, v. 10, n. 19, p. 1058-1078, 2014.

ALVARES, C. A.; STAPE, J. L.; SENTELHAS, P. C.; GONÇALVES, J. L. M.; SPAROVEK, G. Koppen's climate classification map for Brazil. Meteorologische Zeitschrift, v. 22, n. 6, p. 711-728, 2013.

ARRUDA, R.; FADINI, R. F.; CARVALHO, L. N.; DEL-CLARO, K.; MOURÃO, F.A.; JACOBI, C. M., TEODORO, G. S.; VAN DEN BERG, E.; CAIRES, C. S.; DETTKE, G. A. Ecology of neotropical mistletoes: an important canopy- dwelling component of Brazilian ecosystems. Acta Botanica Brasilica, v. 26, p. 264-274, 2012.

ARRUDA, R.; FADINI, R. F.; CARVALHO, L. N.; DEL-CLARO, K.; MOURÃO, F.A.; JACOBI, C. M.; TEODORO, G. S.; VAN DEN BERG, E.; CAIRES, C. S.; DETTKE, G. A. Ecology of neotropical mistletoes: an important canopy-dwelling component of Brazilian ecosystems. Acta Botanica Brasilica, v. 26, n. 2, p. 264-274. 2012.

CAIRES, C. S. Estudos taxonômicos aprofundados de Oryctanthus (Griseb.) Eichler, Oryctina Tiegh. e Pusillanthus Kuijt (Loranthaceae). Tese (Doutorado em Botânica). Universidade de Brasília. Brasília, 2012.

CAIRES, C. S.; UCHÔA-FERNANDES, M. A.; NICÁSIO, J.; STRIKIS, P. C. Frugivoria de larvas de Neosilba McAlpine (Diptera, Lonchaeidae) sobre Psittacanthus plagiophyllus Eichler (Santalales, Loranthaceae) no sudoeste de Mato Grosso do Sul, Brasil. Revista Brasileira de Entomologia, v.02, n. 53, p. 272-277, 2009. 
CAMARGO, N. F.; CRUZ, R. M. S.; RIBEIRO, J. F.; VIEIRA, E. M. Frugivoria e potencial dispersão de sementes pelo marsupial Gracilinanus agilis (Didelphidae: Didelphimorphia) em áreas de Cerrado no Brasil central. Acta Botanica Brasilica, v. 25, n. 3, p. 646-656. 2011.

CARNIELLO, M. A.; LIMA, A. M.; MUNIS, C. C.; DANTAS, T. C. V.; CASTELAN, E.; PEREIRA, M. A.; SILVA, R. A. S.; BELTRAN, M. J. As Loranthaceae e suas hospedeiras no perímetro urbano do Município de Cáceres-MT. In: REUNIÃO ESPECIAL DA SBPC - Mato Grosso: Novos Caminhos - Ambiente e Diversidade Sócio-Cultural,1995. Cuiabá-MT. Anais da II Reunião Especial da SBPC Sociedade Brasileira para o Progresso da Ciência, Cuiabá-MT, p. 556 - 556, 1995.

CHASE, M. W.; REVEAL, J. L. A. An update of the Angiosperm Phylogeny Group classification for the orders and families of flowering plants: APG III. Botanical Journal of the Linnean Society, v. 161, p. 105-121, 2009.

CHIRON, G. R.; PETINI-BENELLI, A. 2012. Une nouvelle espèce d'Acianthera (Orchidaceae, Pleurothallidinae) du Mato Grosso (Brésil). Richardiana, v. 13, p. 6570, 2012.

COONEY, S. J. N.; WATSON, D. M. Diamond firetails (Stagonopleura guttata) preferentially nest in mistletoe. Emu. v. 105, p. 317-322. 2005.

COONEY, S. J. N.; WATSON, D. M.; YOUNG, J. Mistletoe nesting in Australian birds: a review. Emu. v. 106, p. 1-12. 2006.

CRONQUIST, A. The evolution and classification of flowering plants. New York: The New York Botanical Garden, Bronx, 1981. 1262 p.

DAVIDAR, P. Birds and neotropical mistlotoes: effects on seedling recruitment. Oecologia, Berlin, v. 60, p. 271-273, 1983.

DETTKE, G. A.; WAECHER, J. L. Estudo taxonômico das ervas-de-passarinho da Região sul do Brasil: I. Loranthaceae e Santalaceae. Rodriguésia, v. 65, n. 4, p. 939-953, 2014.

DETTKE, G.A.; CAIRES, C.S. Loranthaceae. In: Lista de Espécies da Flora do Brasil. Jardim Botânico do Rio de Janeiro. 2015. Disponível em: <http://floradobrasil.jbrj.gov.br/jabot/floradobrasil/FB152>. Acesso em: 20 ago. 2015

DIBONG, S. D.; ENGONÉ-OBIANG, N. L.; NDONGO, D.; PRISO, R. J.; TAFFOUO, V.; FANKEM, H.; SALLE, G.; MISSOUP, A. D.; BOUSSIM, I. J.; AMOUGOU, A. An assessment on the uses of Loranthaceae in ethno pharmacology in Cameroon: $A$ case study made in Logbessou, North of Douala. Journal of Medicinal Plants Research, v. 3, n. 8, p. 592-595, 2009. 
FERNANDES, J. M.; SOARES-LOPES, C. R. A.; RIBEIRO, R. S.; SILVA, D. R.; Leguminosae no Acervo do herbário da Amazônia Meridional, Alta floresta, Mato Grosso. Enciclopedia Biosfera, v.11, n. 21, p. 2272-2293, 2015.

FIDALGO, O. \& BONONI, V.L.R. Técnicas de coleta, preservação e herborização do material botânico. São Paulo: Instituto de Botânica, 1989. 61p.

FORZZA, R.C.; BAUMGRATZ, J. F. A.; BICUDO, C. E. M.; CANHOS, D. A. L.; CARVALHO-JÚNIOR, A. A.; COELHO, M. A. N.; COSTA, A. F.; COSTA, D. P.; HOPKINS, M. G.; LEITMAN, P. M.; LOHMANN, L. G.; LUGHADHA, E. N.; MAIA, L. C.; MARTINELLI, G.; MENEZES, M.; MORIM, M. P.; PEIXOTO, A. L.; PIRANI, J. R.; PRADO, J.; QUEIROZ, L. P.; SOUZA, S.; SOUZA, V. C.; STEHMANN, J. R.; SYLVESTRE, L. S.; WALTER, B. M. T.; ZAPPI, D. C. New Brazilian Floristic List Highlights Conservation Challenges. Bioscience, v. 62, p. 39-45, 2012.

FREIRE, S.M.F.; ANDRADE, K.N.S.; ARAGÃO JR, G.A.; NORONHA, E.P.; SILVA, S.N.; CARTÁGENES, M.S.S.; BORGES, M.O.R.; RIBEIRO, M.N.S.; TORRES, L.M.B.; BORGES, A.C.R. Antiulcerogenic activity of the extracts of Struthanthus marginatus. Revista Brasileira de Farmacognosia. v. 21, n. 6, p. 1089-1095, 2011.

FRISBY, S.; HIND, D.J.N. Ichthyothere sasakiae, (Compositae: Heliantheae: Miliantheae), a new species from the Amazonian campo rupestre of northern Mato Grosso State, Brazil. Kew Bulletin, v. 69, n. 2, p. 9504-9511, 2014.

GARCÍA, D.; RODRÍGUEZ-CABAL, M.A.; AMICO, G.C. Seed dispersal by a frugivorous marsupial shapes the spatial scale of a mistletoe population. Journal of Ecology. v. 97, p. 217-229, 2009.

GUERRA, T. J.; MARINI, M.A. Bird frugivory on Struthanthus concinnus (Loranthaceae) in Southeastern Brazil. Ararajuba, v. 10, n. 2, p. 187 - 192, dez. 2002.

IBGE - INSTITUTO BRASILEIRO DE GEOGRAFIA E ESTATÍSTICA. Manual técnico da vegetação brasileira. 2 ed. Rio de Janeiro: IBGE, 2012.

ICV - INSTITUTO CENTRO DE VIDA. Parque Estadual Cristalino, Alta Floresta, Amazônia Matogrossense: histórico, situação atual e perspectivas. Cuiabá: ICV, 2003, $13 \mathrm{p}$.

IGANCI, J. R. V.; MORIM, M. P. 2012. Coleções botânicas para conservação: um estudo de caso em Abarema Pittier (Leguminosae, Mimosoideae). Revista Brasileira de Biociências, v. 10, n. 2, p. 164-170, 2012.

IVANAUSKAS, N. M.; MONTEIRO, R.; RODRIGUES, R. R. Classificação fitogeográfica das florestas do Alto Rio Xingu. Acta Amazonica, v. 38, n. 3, p. 387 402, 2008.

JUDD, W. S.; CAPHELL, C. S.; KELLOGG, E. A.; STEVENS, P. F.; DONOGHUE, M. J. Sistemática Vegetal: Um enfoque filogenético. $3^{\underline{a}}$ ed. Tradução de André Olmos 
Simões; Rodrigo B. Singer; Rosana Farias Singer; Tatiana Teixeira de Souza Chies. Porto Alegre: Artmed, 2009. 632 p.

LEAL, L.; BUJOKAS, W.M.; BIONDI, D. Análise da infestação de erva-de-passarinho na arborização de ruas de Curitiba, PR. Floresta. Curitiba, v. 36, n. 3, p. 323-330, 2006.

MARTÍNEZ DEL RIO, C.; SILVA, A.; MEDEL, R.; HOURDEQUIN, M. Seed dispersers as disease vectors: bird transmission of mistletoe seeds to plant hosts. Ecology. v. 77, n. 3, p. 912-921. 1996.

MATHIASEN, R. L.; NICKRENT, D. L.; SHAW, D. C.; WATSON, D. M. Mitletoes: Pathology, systematics, ecology and management. Plant Disease, v. 92, p. 9881006, 2008.

NICKRENT, D. L. Santalales (including mistletoes). In: Encyclopedia of Life Sciences (ELS). John Wiley e Sons, Ltd: Chichester, p. 1-6, 2011.

NICKRENT, D. L.; MALÉCOT, V.; VIDAL-RUSSELL, R.; DER, J. P. A revised classification of Santalales. Taxon. v. 59, n. 2, p. 538-558. 2010.

PENNINGTON, T. D.; CLAKSON, J. J. A revision of Guarea (Meliaceae). Edinburgh Journal of Botany, v. 70, n. 2, p. 179-362, 2013.

PEREIRA, J. L. Prevalência de infecção e padrões de uso de hospedeiros por ervas-de-passarinho (Loranthaceae e Viscaceae) em espécies de árvores tropicais de interesse madeireiro. Dissertação (Mestrado em Recursos Naturais da Amazônia) Universidade Federal do Oeste do Pará. Santarém, 2014.

PETINI-BENELLI, A.; SOARES-LOPES, C.R.A. A new species of Catasetum (Cymbideae, Epidendroideae, Orchidaceae) from the Southern region of the Brazilian Amazon. Phytotaxa, v. 204, n. 1, p. 075-079, 2015.

PETINI-BENELLI, A.; SOARES-LOPES, C.R.A.; SILVA, D.R.; RIBEIRO, R.S. Novos registros de epífitas vasculares para o Estado de Mato Grosso, Brasil. Enciclopédia Biosfera, v.11, n.21; p. 2340-2351, 2015.

RIZZINI, C.T. Pars specialis prodromi monographiae Loranthacearum Brasiliae terrarumque finitimarum. Rodriguésia, v. 18/19, n. 30/31, p. 87-234, 1956.

RODRIGUES, L.; CARVALHO, M. A. C.; SOARES-LOPES, C. R. A.; ROCHAFILHO, J. A.; SANTOS, S. K. F. S. Análise multitemporal da dinâmica de fragmentação no assentamento Arumã, região de influência do Parque Nacional do Juruena, Mato Grosso. Enciclopédia Biosfera, v.10, n.19, p. 1988-2001, 2014.

ROTTA, E. Autotrofia de Tripodanthus acutifolius (Ruiz e Pav.) Thiegh. (erva-depassarinho) um registro. Comunicado Técnico, v. 115, p. 1-3, 2004. 
SANTANA, E. R. D.; MORRESQUE, L. M.; DINIZ, S.; NETO, L. A.; MONTEIRO, D. S.; TAQUES, L. P. Seleção de forófitos pelas ervas de passarinho (Psitacanthus acinarium, Phoradendron rubrum e Phoradendron crassifolium) no parque Zoobotânico da UFMT, Cuiabá. Anais do VIII Congresso de Ecologia do Brasil, Caxambu-MG, 23 a 28 de setembro de 2007.

SANTOS-SILVA, R.; CARNIELLO, M. A. A relação dos moradores da cidade de Cáceres com as ervas-de-passarinho existentes em seus quintais: uma abordagem etnobotânica. In: GUARIM-NETO, G.; CARNIELLO, M. A. (Orgs.). Quintais Mato-Grossenses: Espaços de conservação e reprodução de saberes. Cáceres: UNEMAT, 2008. p. 184-185.

SANTOS-SILVA, R.; CARNIELLO, M. A. Estudo comparado da ocorrência de Loranthaceae no perímetro urbano de Cáceres-MT. Anais do LIII Congresso Nacional De Botânica, Recife-PE, p. 354 - 354, 2002.

SANTOS-SILVA, R. Estudo comparado da ocorrência de Loranthaceae no perímetro urbano de Cáceres, MT. Trabalho de Conclusão de Curso (Graduação em Ciências Biológicas). Universidade do Estado de Mato Grosso. Cáceres, 2002.

SANTOS-SILVA, R. Um estudo bibliográfico sobre os aspectos morfológicos, ecológicos, econômicos e etnobotânicos da família Loranthaceae. Anais do VI Encontro de Botânicos do Centro-Oeste, Cáceres-MT, 2001.

SASAKI, D.; ZAPPI, D.; MILLIKEN, W.; HENICKA, G. S.; PIVA, J. H. Vegetação e Plantas do Cristalino: um manual. Alta Floresta: Royal Botanic Gardens, KEW / Fundação Ecológica Cristalino, 2010. 128p.

SEPLAN. Secretaria de Estado de Planejamento e Coordenação Geral, Perfil do Município. Disponível em: http://www.seplan.mt.gov.br/ Acesso em: 14 de fev., 2014.

SILVA, D. R.; CARNIELLO, M. A.; CAIRES, C. S. "Ervas de Passarinho" ocorrentes na Bacia do Alto Paraguai (BAP), Mato Grosso. Anais da II Jornada Cientifica da Unemat, Cáceres-MT, 05 a 06 de Outubro de 2009. Disponível: http://www.unemat.br/eventos/jornada2009/resumos_conic/Simples_00369.pdf

SILVA, D.R. Hemiparasitas e hospedeiras ocorrentes em três gradientes de umidade de um remanescente de cerrado, Pantanal de Cáceres, Mato Grosso. Trabalho de Conclusão de Curso (Graduação em Ciências Biológicas). Universidade do Estado de Mato Grosso. Cáceres, 2009.

SILVA, D.R.; GUARIM-NETO, G. CARNIELLO, M.A. Hemiparasitas ocorrentes em um remanescente de cerrado associado a pastagens nativas no pantanal de Cáceres, Mato Grosso. Anais do LXI Congresso Nacional de Botânica, ManausAM, 05 a 10 de Setembro de 2010 Disponível: http://www.botanica.org.br/trabalhoscientificos/61CNBot/ResumoS01_CNBot_2010_ 317.pdf 
SOARES-LOPES, C. R. A.; RIBEIRO, R. S.; RODRIGUES, L.; CABRAL, F. F.; SILVA, D. R. Check list de Angiospermas da Região de Influência da UHE Sinop, Médio Teles Pires, Mato Grosso. Enciclopédia Biosfera, v.10, n.19, p. 2036-2048, 2014.

SOUZA, V. C.; LORENZI, H. Botânica Sistemática: Guia ilustrado para identificação das famílias de fanerógamas nativas e exóticas no Brasil, baseado em APG III. 3 ed. Nova Odessa: Instituto Plantarum. 2012. 768 p.

SPLETOZER, A. G.; SANTOS, L. G.; VERÃO, D. S.; RODRIGUES, L. SOARESLOPES, C.R.A. Mapeamento das classes de uso e ocupação do solo na Fazenda Nova Vida, Alta Floresta - MT. Enciclopédia Biosfera, v.10, n.19, p. 1272-1279, 2014.

TORRES, F.; MONTILLA, M. Implantación de la Loranthaceae hemiparasita Phthirusa pyrifolia (H.B.K.) Eichl. en especies de un agroecosistema cafetero. Plantula. v. 1, n. 3, p. 213-219, 1997.

VANDERPLANK, J.; ZAPPI, D. Passiflora cristalina, a striking new species of Passiflora (Passifloraceae) from Mato Grosso, Brazil. Kew Bulletin, v. 68, n.1, p. 149-153, 2011.

VICECONTE, K. S. M.; MAIA, V. C. Novo gênero e nova espécie de Diptera, Cecidomyiidae, associada com Psittacanthus dichrous (Loranthaceae). Arquivos do Museu Nacional, v. 7, p. $35-40,2009$.

VIEIRA, O. M. C.; SANTOS, M. H.; SILVA, G. A.; SIQUEIRA, A. M. Atividade antimicrobiana de Struthanthus vulgaris (erva-de-passarinho). Revista Brasileira de Farmacognosia, v. 15, n. 2, p. 149-154, 2005.

WHITE, B. L. A.; RIBEIRO, A. S.; WHITE, L. A. S.; NASCIMENTO-JUNIOR, J. E. Análise da ocorrência de erva-de-passarinho na arborização da Universidade Federal de Sergipe, Campus São Cristóvão. Floresta, v. 41, p. 1-8, 2011.

ZAPPI, D. C.; SASAKI, D.; MILLIKEN, W.; IVA, J.; HENICKA, G. S.; BIGGS, N.; FRISBY, S. Plantas vasculares da região do Parque Estadual Cristalino, Norte de Mato Grosso, Brasil. Acta Amazônica, v. 41, p. 29-38, 2011. 